\title{
Brain Areas Consistently Linked to Individual Differences in Perceptual Decision-making in Younger as well as Older Adults before and after Training
}

\author{
Simone Kühn ${ }^{1,2}$, Florian Schmiedek ${ }^{3,4}$, Björn Schott ${ }^{5,6}$, \\ Roger Ratcliff ${ }^{7}$, Hans-Jochen Heinze ${ }^{5}$, Emrah Düzel ${ }^{2,5}$, \\ Ulman Lindenberger ${ }^{3}$, and Martin Lövden ${ }^{3,8}$
}

\begin{abstract}
Perceptual decision-making performance depends on several cognitive and neural processes. Here, we fit Ratcliff's diffusion model to accuracy data and reaction-time distributions from one numerical and one verbal two-choice perceptual-decision task to deconstruct these performance measures into the rate of evidence accumulation (i.e., drift rate), response criterion setting (i.e., boundary separation), and peripheral aspects of performance (i.e., nondecision time). These theoretical processes are then related to individual differences in brain activation by means of multiple regression. The sample consisted of 24 younger
\end{abstract}

\section{INTRODUCTION}

It has been a fundamental goal of cognitive neuroscience to link cognitive and neural processes. One of the many problems in this endeavor is that global cognitive performance measures are influenced by many underlying processes. For example, the performance measures most commonly used in experimental psychology, mean reaction times for correct decisions (RT) and accuracy, are not only a function of the efficiency of the targeted cognitive processes but also of the subjects' response criterion, as speed and accuracy are in a tradeoff relationship: Hasty decisions are more prone to errors, whereas careful and accurate judgments take more time (Wickelgren, 1977). To deconstruct global cognitive performance measures and, for example, disentangle criterion setting from process efficiency, mathematical models can however be applied. Here, we use Ratcliff's (1978) diffusion model (Ratcliff \& Tuerlinckx, 2002) to disentangle the speed-

\footnotetext{
${ }^{1}$ Ghent University, Belgium, ${ }^{2}$ University College London, UK,

${ }^{3}$ Max Planck Institute for Human Development, Berlin, Germany, ${ }^{4}$ German Institute for International Educational Research, Frankfurt am Main, Germany, ${ }^{5}$ Otto-von-Guericke University Magdeburg, Germany, ${ }^{6}$ Charité University Medecine, Berlin, ${ }^{7}$ Ohio State University, Columbus, ${ }^{8}$ Lund University, Sweden
}

and 15 older adults performing the task in fMRI before and after 100 daily 1-hr behavioral training sessions in a multitude of cognitive tasks. Results showed that individual differences in boundary separation were related to striatal activity, whereas differences in drift rate were related to activity in the inferior parietal lobe. These associations were not significantly modified by adult age or perceptual expertise. We conclude that the striatum is involved in regulating response thresholds, whereas the inferior parietal lobe might represent decision-making evidence related to letters and numbers. accuracy tradeoff (Ratcliff, 2002; Ratcliff \& Rouder, 2000) and to relate individual differences in perceptual decisionmaking processes to underlying brain regions (Bogacz, Wagenmakers, Forstmann, \& Nieuwenhuis, 2010).

The diffusion model aims to explain data from twochoice RT tasks in a comprehensive way, utilizing accuracy information as well as the shape of the RT distribution for correct and erroneous responses. This is achieved by assuming that perceptual-decision behavior involves several processes that come with associated parameters (Figure 1). First, the quality of evidence accumulation during the decision process, the drift rate, is a central parameter. It describes how quickly information is accumulated in a random walk-like diffusion process that progresses from a starting point toward one of two response boundaries, one for correct and one for wrong responses. Higher drift rates indicate faster accumulation of evidence; that is, a more efficient decision process. A second central parameter of the model characterizes response criteria. This more strategic aspect of decision behavior is implemented by differences in the distance between the response boundaries, the boundary separation. Wider boundary separation means more conservative responding because more evidence needs to be accumulated before a boundary is reached and a response is initiated. Still, another 


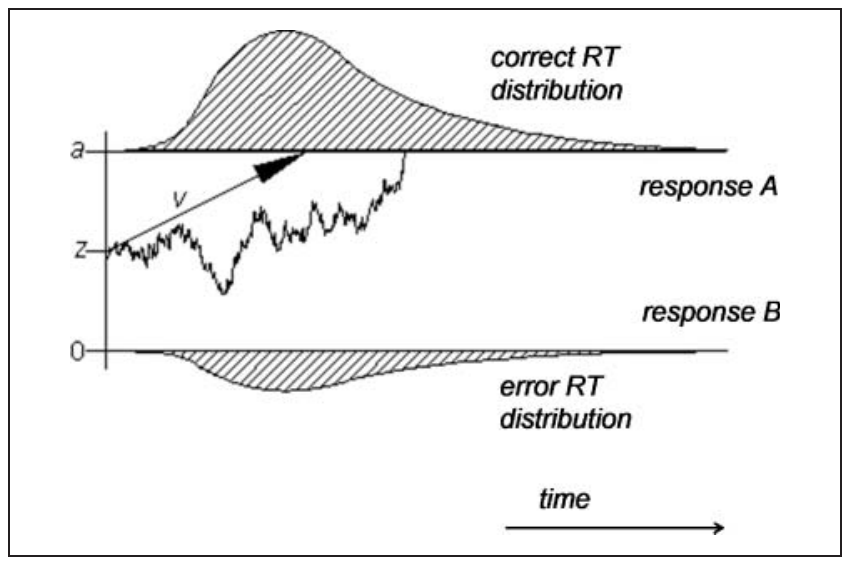

Figure 1. An illustration of the diffusion model with one simulated paths with drift rate $v$, boundary separation $a$, and starting point $z$.

parameter is nondecision time, combining the efficiency of peripheral sensory and motor aspects of the decision process.

In this study, we applied the diffusion model to data from a two-choice decision task with numerical or verbal content. In the numerical task, participants had to judge whether briefly presented numbers were odd or even. In the letter task, participants decided whether a selection of letters were consonants or vowels. Interindividual differences in the estimates of the diffusion-model parameters were then linked to differences in brain activation as observed with functional magnetic resonance imaging (fMRI) during performance of these tasks. In line with the assumptions of the diffusion model and its theoretical application to neuroscience (Heekeren, Marrett, \& Ungerleider, 2008), we hypothesized that the drift rate, indicating perceptual evidence accumulation, should be associated with activation in stimuli-specific brain regions, that is, in regions involved in processing of numbers and letters (Joseph, Cerullo, Farley, Steinmetz, \& Mier, 2006; James, James, Jobard, Wong, \& Gauthier, 2005; Dehaene, Piazza, Pinel, \& Cohen, 2003; Eger, Sterzer, Russ, Giraud, \& Kleinschmidt, 2003; Joseph, Gathers, \& Piper, 2003), such as the left inferior parietal lobe. This prediction is, for example, supported by studies on monkeys that have modeled neurophysiological data from intracranial recordings as diffusion processes (Ratcliff, Hasegawa, Hasegawa, Smith, \& Segraves, 2007; Ratcliff, Cherian, \& Segraves, 2003; Kim \& Shadlen, 1999). In Kim and Shadlen's task, monkeys had to perform a direction of motion visual discrimination task while data were recorded in cells downstream of area MT (lateral intraparietal area, frontal eye fields, superior colliculus, and dorsolateral prefrontal cortex). Their results suggest that decisions are formed by computing the difference between the activities of populations of neurons in area MT that code for opposite directions of motion, and this decision variable is represented in the downstream cells. The results from a large number of studies support the view that decision-making is based on the integration of noisy sensory evidence that is repre- sented by sensory neurons (e.g., Smith \& Ratcliff, 2004; Gold \& Shadlen, 2001). For the boundary parameter, we predicted, based on previous studies focussing on the speed-accuracy tradeoff, an association with activity in the striatum and the presupplementary motor area (Forstmann et al., 2008; Ivanoff, Branning, \& Marois, 2008; Van Veen, Krug, \& Carter, 2008). The basal ganglia (striatum and pallidum) may implement a generic action-selection mechanism that releases those actions from inhibition that are desirable and maintains inhibitory control over others (Mink, 1996), thereby acting as a gate-keeper (see also Frank, 2006). Thus, it is plausible to assume that activity in the striatum is related to criterion setting.

To address these hypotheses, we utilized data from a large-scale study (COGITO Study; Schmiedek, Bauer, Lövdén, Brose, \& Lindenberger, 2010; Schmiedek, Lövdén, \& Lindenberger, 2010) including younger and older participants performing six tests of perceptual speed (including the two tasks reported in the present article), three tests of episodic memory, and three tests of working memory in approximately 100 daily 1 -hr sessions. Before and after the 100-day-long longitudinal phase, extensive data on two-choice RT performance were collected, allowing for estimation of the diffusion parameters as well as for investigating age differences and practice-related changes in these parameters. In this article, we report data for a subsample of participants that additionally performed the two-choice RT tasks in the MRI scanner at pretest and posttest.

Previous studies on age differences in diffusion-model parameters have primarily reported that more conservative response criteria in old age as well as age-related slowing of nondecision time contribute to age-related slowing of perceptual decision making (Starns \& Ratcliff, 2010; Ratcliff, Thapar, \& McKoon, 2001, 2006; Ratcliff, Thapar, Gomez, \& McKoon, 2004; Thapar, Ratcliff, \& McKoon, 2003). Practice has been reported to result in faster evidence accumulation and less conservative response criteria, with older adults changing more in these parameter estimates (Ratcliff et al., 2006). fMRI studies of activation changes as a function of practice on lower-level sensory/ motor tasks, in contrast to higher-level cognitive tasks such as working memory, have demonstrated decrease of activity in task-related areas after practice (Kelly \& Garavan, 2005). In addition, activity in brain areas that have been associated with the so-called resting-state or default-mode (Raichle et al., 2001) network increase during task performance after practice (Mason et al., 2007). This pattern likely reflects more automatic, less attention-demanding, execution of the task posttraining (Kelly \& Garavan, 2005). Thus, the data reported in this study allow for examining the generality of the predicted link between regional brain activation and aspects of perceptual decision-making behavior at different average levels of global performance and of the underlying processing components, across younger and older adults, and for nonexpert and highly skilled, less effortful, execution of the task. 


\section{METHODS}

\section{Participants}

A healthy subsample consisting of younger $\left(n=24 ; M_{\text {age }}=\right.$ 25.2 years, $S D_{\text {age }}=3.2$, range $_{\text {age }}=20.5-31.1$ years $)$ and older $\left(n=15 ; M_{\text {age }}=70.2\right.$ years; $S D_{\text {age }}=4.0$, range $_{\text {age }}=$ 65.0-80.4 years) adults was recruited for imaging from a larger study on day-to-day variability and plasticity of cognitive performance (the COGITO Study; Schmiedek, Bauer, et al., 2010; Schmiedek, Lövdén, et al., 2010), which originally recruited participants through newspaper advertisements, word-of-mouth recommendation, and flyers circulated in Berlin, Germany. All participants of this parent study were asked to take part in imaging, and those volunteering were screened for eligibility. Accepted participants were right-handed, had normal or corrected-tonormal vision, and reported no history of cardiovascular disease (except treated hypertension), diabetes, neurological or psychiatric conditions, or drug/alcohol abuse. They reported no use of antiseizure or antidepressant drugs. Finally, considered participants completed pretest imaging, cognitive training, and posttest imaging without producing imaging artifacts or displaying brain abnormalities. In terms of global cognitive performance measures [Digit-Symbol Substitution (DS) and Vocabulary], selectivity $\left[\left(M_{\text {Imaging Sample }}-M_{\text {Total Sample }}\right) / S D_{\text {Total Sample }}\right]$ was negligible for younger adults $(S D=0.05$ for DS and $S D=0.00$ for vocabulary). As expected from the contraindications for MRI, the older MRI sample was positively selected $(S D=1.01$ for DS and $S D=0.73$ for vocabulary).

Participants were paid between 1450 and 1950 Euros, depending on the number of completed sessions and their pace of completing them. All participants gave written informed consent. The ethical review board of the Otto-vonGuericke University of Magdeburg approved the imaging study and the review board of the Max Planck Institute for Human Development, Berlin, approved the behavioral parent study.

\section{Materials and Procedure}

\section{Training Phase}

Participants practiced computerized tasks individually during, on average, $101\left(M_{\text {young }}=102, S D_{\text {young }}=3.1 ; M_{\text {old }}=\right.$ 99, $S D_{\text {old }}=3.7$ ) sessions. Practiced tasks were administered with several stimuli-presentation times at pretest, which allowed for estimation of time-accuracy functions used for tailoring subsequent task difficulty by adjusting presentation times for each individual at the start of training. Presentation times were subsequently kept constant. Working memory tasks used included 3-back, numerical updating, and alpha span. Episodic memory tasks used included word list recall, object-location memory, and number-noun pairs. Speed tasks were three comparison and three two-choice RT tasks. Each ability domain contained tasks with numerical, verbal, and figural-spatial content. Schmiedek, Bauer, et al. (2010) and Schmiedek,
Lövdén, et al. (2010) describe these tasks in detail. Here we describe the two-choice RT tasks reported in this article in more detail.

The three two-choice RT tasks were based on the same stimulus layout, the seven lines of the number " 8 " as displayed on pocket calculators (Figure 2). Stimuli were masked with a stimulus that combined this "calculator 8" with extending lines in all 10 possible directions. Masking times used at pre- and posttest were 1, 2, 4, and 8 screen cycles $(12,24,47$, and $94 \mathrm{msec})$ for younger and 2, 4, 8, and 16 screen cycles $(24,47,94$, and $188 \mathrm{msec})$ for older adults. Possible masking times for the daily sessions were 1, 2, 4, or 8 screen cycles. Depending on pretest performance, two of these masking times (one fast and one slow condition) were chosen for each participant to work on during the training phase, in the following way. For each two-choice task and each individual, mean accuracies for the different masking time conditions at pretest were fitted with exponential time-accuracy functions (including freely estimated parameters for onset, rate, and asymptote as well as a lower asymptote parameter fixed to 0.50). The fitted values from these functions were used to choose one "fast" and one "slow" masking time, which were both chosen so that the predicted accuracy based on the time-accuracy functions was above a minimum level and below an upper level. If performance was above the upper level for the second-but-fastest masking time, then the fastest masking time was chosen, even if predicted accuracy was below the minimum level for the fastest masking time. The fast masking time was chosen based on a minimum level of 0.625 and an upper level of 0.75 , whereas for the slow masking time, those levels were 0.875 and 0.95 , respectively.

Each two-choice RT block consisted of 40 stimuli, 20 for the fast and 20 for the slow condition, with randomly

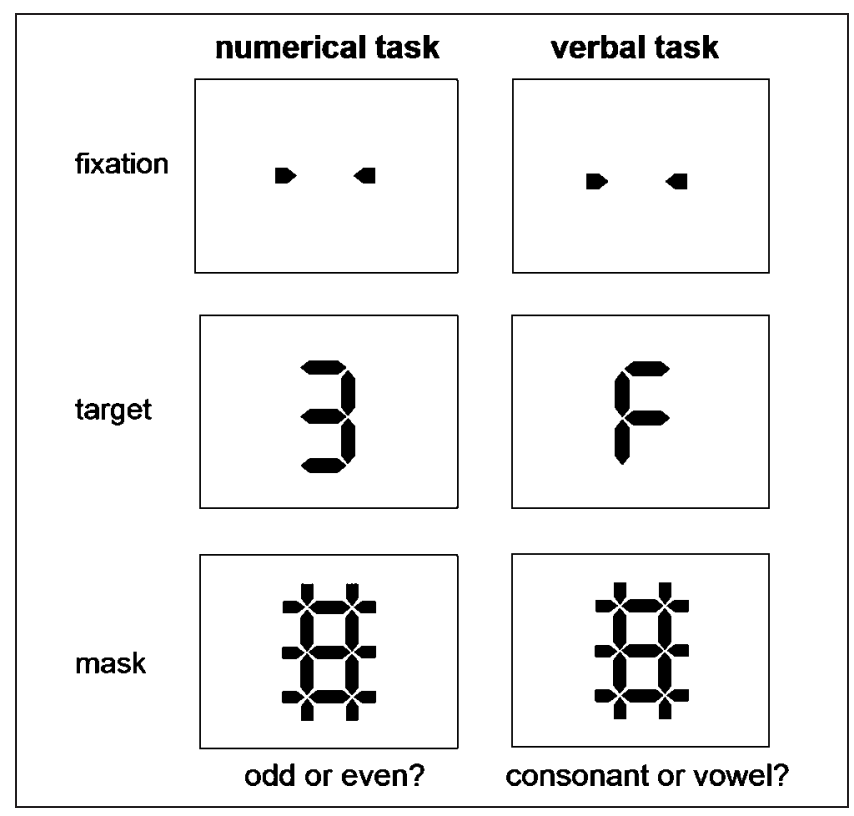

Figure 2. Schematic drawing of the experimental paradigm. 
chosen stimuli out of the two response categories. In the figural task, participants decided whether the stimuli were either symmetric or asymmetric to stimuli that were either the upper or lower two lines to the left and right of the "calculator 8" (symmetric condition), or the two possible combinations of one upper and one lower line at the left and right (asymmetric condition). In the numerical task, participants decided whether the displayed number was odd or even. Stimuli were "3," "5," and "7," for the odd condition and " 2 ," " 4 ," and " 6 " for the even condition. In the verbal task, participants decided whether letters were either consonants or vowels. Stimuli were "F," "H," and "P" for the consonant condition and "A," "E," and "U" for the vowel condition. No performance feedback was provided during the task.

\section{Pretest and Posttest}

Participants completed behavioral pretests and posttests during 10 sessions that consisted of 2 to $2.5 \mathrm{hr}$ of cognitive test batteries and self-report questionnaires. The pretest brain imaging session was conducted after the behavioral pretest and immediately before the longitudinal practice phase. The posttest imaging session was completed shortly after the completion of the behavioral posttest. The sessions were separated by an average of 179 days $\left(M_{\text {young }}=180, S D_{\text {young }}=21.0 ; M_{\text {old }}=179\right.$, $S D_{\text {old }}=31.1$ ). Below we describe the assessment of the two-choice RT performance in more detail.

Behavioral assessment of two-choice RT data. Performance on the two-choice RT tasks was assessed within one behavioral session of about 2.0 to $2.5 \mathrm{hr}$ at pretest and again at posttest. In these sessions, participants worked on the verbal, numerical, and figural versions of the task. Tasks and stimuli were identical for pretest and posttest. In each session, participants worked on 25 blocks consisting of 40 items each for each task, with breaks after sets of 5 blocks (length of break determined by participants). Each block consisted of 20 odd/consonant/symmetric and 20 even/vowel/asymmetric stimuli. Masking times were distributed randomly, but with equal frequencies (10 items per masking time) across blocks, resulting in a total of 250 items per masking time and task. At pretest, participants additionally did first work on two blocks of each task without masking and then on another three practice blocks. These blocks were not used in the current analyses. Participants responded on a customized button box connected to the parallel port for high resolution of RT measurements.

The diffusion model was fit to the data for each task and each subject by minimizing a Chi-square value with a general SIMPLEX minimization routine that adjusts the parameters of the model until it finds the parameter estimates that minimize the Chi-square value (see Ratcliff \& Tuerlinckx, 2002, for a full description of the method). For each experimental condition, the $0.1,0.3,0.5,0.7,0.9$ quantile RTs for correct and error responses and the corresponding accuracy values were entered into the minimization routine. The quantile RTs and the diffusion model were used to generate the predicted cumulative probability of a response as a function of quantile RTs. By subtracting the cumulative probabilities for each successive quantile from the next higher quantile, one gets the proportion of responses between adjacent quantiles. These are the expected values to be compared to the observed proportions of responses between the quantiles (i.e., the proportions between $0,0.1,0.3,0.5,0.7,0.9$, and 1.0 , which are $0.1,0.2,0.2,0.2,0.2$, and 0.1 ) multiplied by the number of observations for the Chi-square computation. Summing over (Observed - Expected $)^{2} /$ Expected for all conditions results in a single Chi-square value to be minimized. Estimated parameters were drift rate, boundary separation, nondecision time, across-trial variability in drift rate (normally distributed), across-trial variability in starting point (uniformly distributed), across-trial variability in nondecision time (uniformly distributed), and the probability of contaminant responses stemming from a uniform distribution. Drift rates were allowed to vary across masking time conditions. For the analyses reported here, only the drift rate estimates for the conditions with masking time of four screen cycles were used, as those matched the masking time in the scanner session. All other parameters were assumed to be invariant across masking time conditions. As shown in Figure 3, plotting characteristics of observed data versus theoretical expectations based on estimated parameters indicated that the model did fit the data quite well with very few individuals showing deviations. No alternative models were tested, so there was no model selection.

Behavioral task in MRI scanner. The task consisted of 53-sec-long task blocks alternating between the verbal and the numerical tasks described above, intermixed with 16-sec fixation blocks. Each task block included 16 trials (8 odd/8 even; 8 consonants/8 vowels) presented with jittered interstimulus intervals between 2000 and 8000 msec. In total, eight blocks per task were presented, distributed over four runs. Thus, in total, 128 trials per task were presented, with randomly chosen stimuli out of the response categories. Masking times were $50 \mathrm{msec}$ for all participants and assessments.

MRI procedures. Images were collected with a 3-T Magnetom Trio MRI scanner system (Siemens Medical Systems, Erlangen, Germany). Whole-brain functional images were collected using a T2*-weighted EPI sequence sensitive to BOLD contrast $(\mathrm{TR}=2000 \mathrm{msec}, \mathrm{TE}=30 \mathrm{msec}$, image matrix $=64 \times 64, \mathrm{FOV}=224 \mathrm{~mm}$, flip angle $=80^{\circ}$, slice thickness $=3.5 \mathrm{~mm}$, distance factor $=0 \%$, voxel size $3.5 \times 3.5 \times 3.5 \mathrm{~mm}^{3}, 32$ axial slices). One hundred fortyseven image volumes were acquired per run all aligned to AC-PC. Anatomical images were acquired using a T1weighted sagittal 3-D spoiled gradient-echo (SPGR) image 


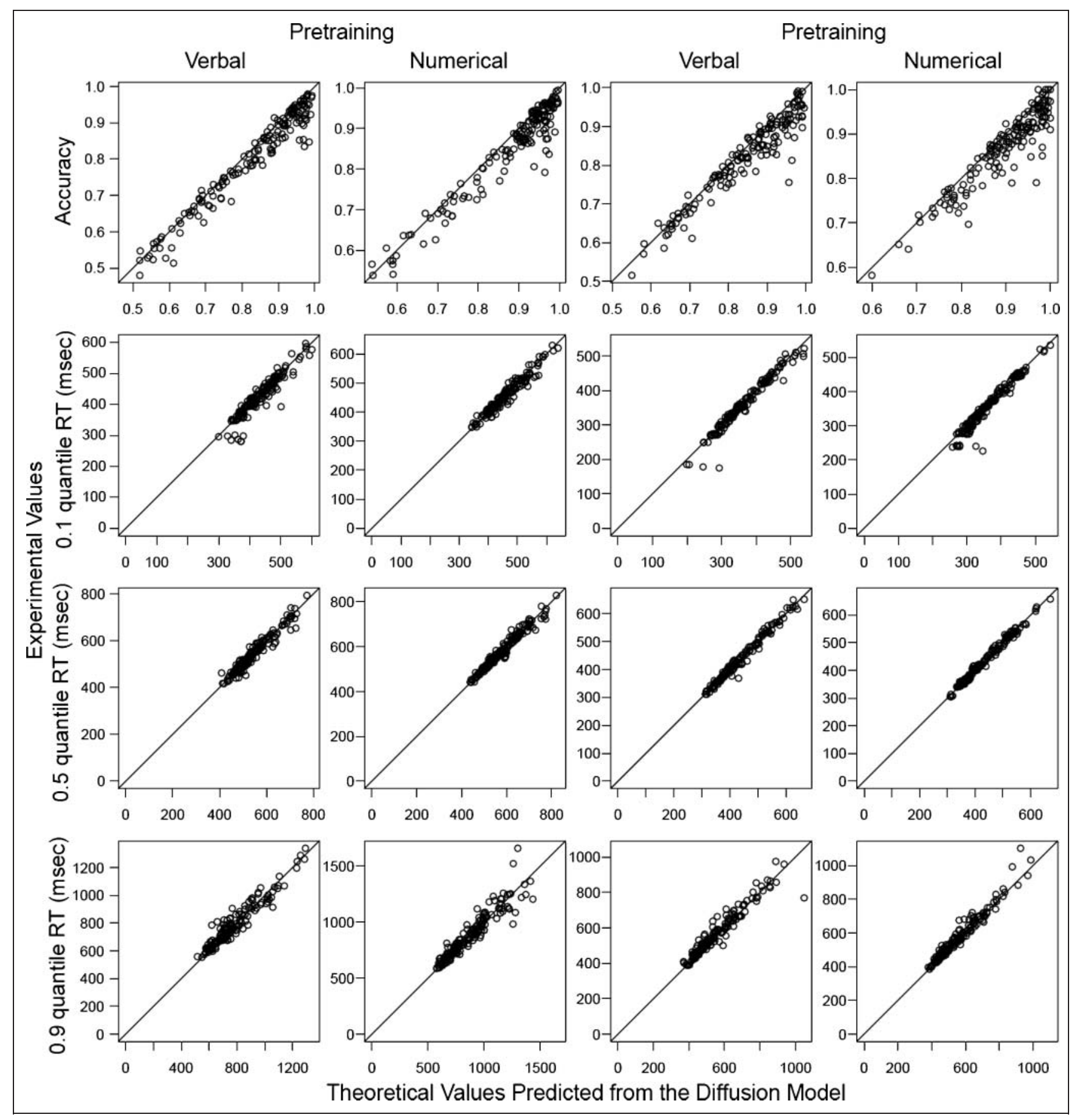

Figure 3. Plots of accuracy and RT quantiles for data ( $y$-axis) and predicted values from fits of the diffusion model ( $x$-axis).

$(\mathrm{TR}=24 \mathrm{msec}, \mathrm{TE}=8 \mathrm{msec}$, acquisition matrix $=256 \times$ $256 \times 124, \mathrm{FOV}=250 \times 250 \mathrm{~mm}^{2}$, flip angle $=30^{\circ}$, slice thickness $=1.5 \mathrm{~mm}$ ) on a GE Signa system (General Electric, Milwaukee, WI).

\section{fMRI Data Analysis}

Preprocessing. The fMRI data were analyzed using the SPM5 software (Wellcome Department of Cognitive Neurology, London, UK). The first three volumes of all EPI series were excluded from the analysis to allow the magnetization to approach a dynamic equilibrium. Data processing started with slice time correction and realignment of the EPI datasets. A mean image for all EPI volumes was created, to which individual volumes were spatially realigned by means of rigid-body transformations. The high-resolution structural image was coregistered with the mean image of the EPI series. Then the structural image was normalized to the Montreal Neurological Institute (MNI) template, and the normalization parameters were applied to the EPI images 
to ensure an anatomically informed normalization. A commonly applied filter of $8 \mathrm{~mm}$ full width at half maximum was used. Low-frequency drifts in the time domain were removed by modeling the time series for each voxel by a set of discrete cosine functions to which a cutoff of $224 \mathrm{sec}$ was applied.

Statistical analyses. The single-subject-level statistical analyses were performed using a general linear model. We modeled the stimulus onset of the numerical and verbal stimuli. Vectors containing the event onsets were convolved with the canonical hemodynamic response function to form the main regressors in the design matrix (the regression model). The vectors were also convolved with the temporal derivatives and the resulting vectors were entered into the model. In addition, the design matrix included the six realignment parameters to further correct for head motion. The statistical parameter estimates were computed separately for each voxel for all columns in the design matrix. Contrast $(t)$ images were constructed from each individual to compare the relevant parameter estimates for the regressors containing the canonical hemodynamic response function.

Next, group-level random effects analysis was performed. A full-factorial ANOVA with the within-subject factors task (numerical vs. verbal) and time point (pretraining vs. posttraining) and the between-subject factor age (young vs. old) was performed for each voxel of the contrast images. The resulting statistical values of the $t$ contrasts were thresholded with $p<.05$ (family-wise error corrected) and a cluster threshold of 5 adjacent voxels.

Finally, we combined the results from the mathematical modeling and the fMRI measurements in order to explore the neural correlates of the drift rate, boundary separation, and nondecision time parameters. To do so, we correlated the diffusion-model parameters derived for each individual with the task-related percent signal change of the whole brain (verbal task $>$ baseline, numerical task $>$ baseline) by means of a multiple regression analysis that included dummy-coded regressors for task, age, and time point, as well at the parameters of drift rate, boundary separation, and nondecision time. The resulting statistical values were thresholded with $p<.05$ (family-wise error corrected) and a cluster threshold of 5 adjacent voxels. To address the generality of observed effects across age and time point, we next included the interaction terms between age and time point on the one hand and the diffusion-model parameters on the other into the multiple regression. To explore the task-specific effects of drift rate, we lowered the threshold to $p<.001$ (uncorrected) with a cluster threshold of at least 10 adjacent voxels. All resulting statistical maps were overlaid onto a normalized T1-weighted MNI singlesubject template (colin27).

In order to extract percent signal changes from the activated clusters of interest, we used MarsBaR (http://marsbar. sourceforge.net/, Brett, Anton, Valabregue, \& Poline, 2002).
For each subject, region, and condition separately, the mean percent signal change over a time window of 4-6 sec after stimulus onset was computed.

\section{RESULTS \\ Behavioral Data}

\section{Accuracy and RT during MRI}

A repeated measures ANOVA on accuracy with the factors Task (numerical vs. verbal), Time point (pretraining vs. posttraining), and Age group (young vs. old) revealed a significant main effect of Time point $[F(1,37)=112.86, p<.001]$, reflecting a general improvement over time; a significant main effect of Age $[F(1,37)=17.12, p<.001]$, revealing higher performance for younger adults; and a significant main effect of Task $[F(1,37)=77.17, p<.001]$, indicating that the accuracy was higher in the numerical compared to the verbal task. Moreover, we found a significant Task $x$ Age interaction $[F(1,37)=4.30, p<.05]$, indicating a higher accuracy difference between the two tasks for older adults; a Time point $\times$ Age interaction $[F(1,37)=14.41, p<.01]$, reflecting a stronger increase in accuracy over time for older adults; and a Time point $\times$ Task interaction $[F(1,37)=$ $22.06, p<.001]$, revealing that task differences in accuracy were reduced after training.

The analysis of RTs revealed a significant main effect of Time point $[F(1,37)=53.66, p<.001]$, reflecting a general improvement over time, and a significant main effect of Age $[F(1,37)=5.58, p<.05]$, revealing faster responses for younger adults. Moreover, we found a significant threefold Task $\times$ Time point $\times$ Age interaction $[F(1$, $37)=4.67, p<.05]$, reflecting a stronger interaction of Task and Age group in the pretraining session compared to the posttraining session (Table 1).

\section{Parameter Estimates of the Diffusion Model}

We performed a separate repeated measures ANOVA on the estimates of the diffusion-model parameters (Table 1) of the data acquired outside the scanner using the same task with the factors Task (numerical vs. verbal), Time point (pretraining vs. posttraining), and Age group (young vs. old).

For the drift rate, we found a significant main effect of Task $[F(1,37)=42.48, p<.001]$, indicating that the drift rate was higher in the numerical compared to the verbal task; and a significant effect of Time point $[F(1,37)=$ $10.59, p<.05]$, reflecting a general improvement over time. Moreover, we detected a significant Time point $\times$ Age interaction $[F(1,37)=4.76, p<.05]$, with a stronger increase in drift rates for the younger compared to the older adults. The threefold interaction of Task $\times$ Time point $\times$ Age was also significant $[F(1,37)=28.91, p<.001]$, indicating larger improvements over time for older adults in the numerical task, which contrast with the larger improvements in the verbal task for the younger adults. No significant main effect of Age group was found. 
Table 1. Mean and Standard Deviation of RT and Accuracy from Task Performance in the Scanner

\begin{tabular}{|c|c|c|c|c|c|c|c|c|}
\hline & \multicolumn{2}{|c|}{ Young Numerical Task } & \multicolumn{2}{|c|}{ Young Verbal Task } & \multicolumn{2}{|c|}{ Old Numerical Task } & \multicolumn{2}{|c|}{ Old Verbal Task } \\
\hline & Pretest & Posttest & Pretest & Posttest & Pretest & Posttest & Pretest & Posttest \\
\hline RTs (msec) & $742(123)$ & $584(107)$ & $793(130)$ & $593(99)$ & 824 (169) & $697(94)$ & 814 (208) & $710(95)$ \\
\hline Accuracy & $0.89(0.116)$ & $0.94(0.068)$ & $0.74(0.133)$ & $0.91(0.062)$ & $0.73(0.148)$ & $0.93(0.060)$ & $0.55(0.110)$ & $0.83(0.141)$ \\
\hline Drift rate & $0.351(0.114)$ & $0.406(0.133)$ & $0.230(0.116)$ & $0.317(0.121)$ & $0.430(0.132)$ & $0.578(0.171)$ & $0.465(0.124)$ & $0.497(0.159)$ \\
\hline Boundary & $0.159(0.030)$ & $0.083(0.022)$ & $0.116(0.029)$ & $0.075(0.015)$ & $0.124(0.031)$ & $0.116(0.080)$ & $0.121(0.032)$ & $0.097(0.024)$ \\
\hline Nondecision & $0.401(0.039)$ & $0.310(0.031)$ & $0.386(0.035)$ & $0.316(0.033)$ & $0.461(0.050)$ & $0.403(0.062)$ & $0.466(0.048)$ & $0.423(0.051)$ \\
\hline
\end{tabular}

Diffusion-model parameters from behavioral assessment outside the scanner.

The boundary parameter revealed a significant main effect of Task $[F(1,37)=15.35, p<.001]$, with a stricter decision criterion for the numerical compared to the verbal task, and a significant effect of Time point $[F(1,37)=$ $36.62, p<.001]$, reflecting a general reduction in strictness of the boundary over time. Moreover, we found a significant Time point $\times$ Age interaction $[F(1,37)=$ $11.80, p<.05]$, with a stronger decline in the boundary parameter for the younger compared to the older adults. The threefold interaction of Task $\times$ Time point $\times$ Age was again significant $[F(1,37)=8.01, p<.05]$, indicating larger decreases over time for younger adults in the numerical task, which contrast with the larger decreases in the verbal task for the older adults. No significant main effect of Age group was found.

The nondecision parameter revealed a significant main effect of Time point $[F(1,37)=154.40, p<.001]$, reflecting a general reduction in nondecision time, and a significant main effect of Age $[F(1,37)=55.70, p<.001]$, indicating shorter nondecision times in the young. Moreover, we found a significant Time point $\times$ Age interaction $[F(1,37)=8.21, p<.01]$, with a stronger reduction of nondecision time for the younger compared to the older adults.

\section{fMRI Data}

\section{Full-factorial ANOVA}

A random effects analysis of the factor Time point from the full-factorial ANOVA containing the factors Task (numerical vs. verbal) and Time point (pretraining vs. posttraining) and the factor Age (young vs. old) revealed significantly more activity at pretest compared to posttest in brain areas that have been associated with the so-called cognitive control network. In detail, we found higher pretest compared to posttest activity in dorsal anterior cingulate cortex (dACC), bilateral insular cortex, left inferior frontal junction (IFJ), left inferior frontal gyrus triangularis (IFG), and right fusiform gyrus (Figure 4A and Table 2A). Increased activity at posttest compared to pretest was found in brain areas that have been associated with the so-called default mode network, namely, ventromedial prefrontal cortex
(vmPFC) and the precuneus, as well as the right superior temporal gyrus (STG) (Figure 4B and Table 2B).

\section{Multiple Regression Analysis with Diffusion-model Parameters}

Next, we examined the predictive effects of the diffusionmodel parameters on the whole-brain activation maps in a multiple regression model also including the factors Task, Age, and Time point as predictors.

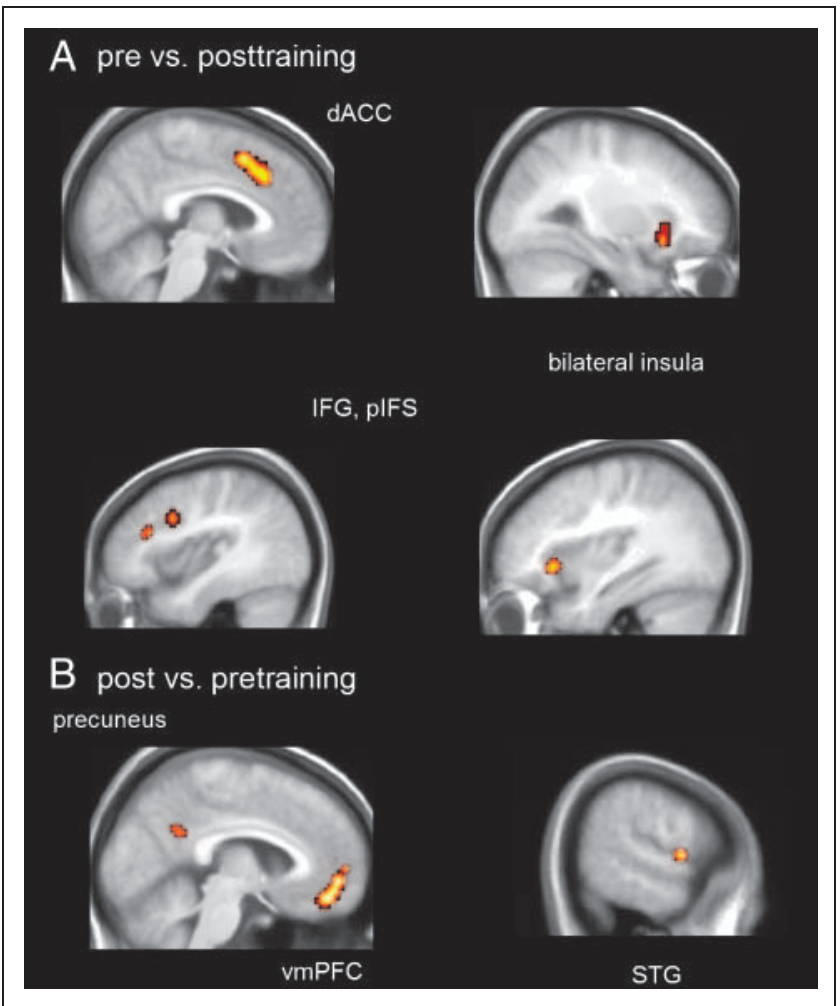

Figure 4. Activation map averaged over 39 subjects $(p<.05$, family-wise error corrected, $k>5$ ) mapped onto an averaged image of the participants' normalized (MNI) T1-weighted images. (A) Significantly higher brain activity in the pretraining compared to the posttraining scanning session; (B) Significantly higher brain activity in the posttraining compared to the pretraining scanning session. 
Table 2. Brain Areas Showing Significant Differences between Pre- and Posttest

\begin{tabular}{|c|c|c|c|c|}
\hline Area & $B A$ & Peak Coordinates (MNI) & Z-score & Extent \\
\hline \multicolumn{5}{|l|}{ (A) Pretraining vs. Posttraining } \\
\hline Rostral cingulate zone & 32 & $-6,18,45$ & 6.85 & 188 \\
\hline Left insular cortex, inferior frontal gyrus & 47 & $-30,21,-9$ & 5.53 & 26 \\
\hline Right fusiform gyrus & 19 & $27,-66,-6$ & 5.41 & 14 \\
\hline Left inferior frontal junction & 44 & $-42,9,33$ & 5.39 & 13 \\
\hline Right insular cortex, inferior frontal gyrus & 47 & $33,24,-3$ & 4.94 & 20 \\
\hline Left inferior frontal gyrus triangularis & 45 & $-39,27,21$ & 4.93 & 7 \\
\hline \multicolumn{5}{|l|}{ (B) Posttraining vs. Pretraining } \\
\hline Ventromedial prefrontal cortex & $10 / 11$ & $-3,51,-15$ & 5.44 & 52 \\
\hline Right superior temporal gyrus & 22 & $60,3,3$ & 5.21 & 9 \\
\hline Precuneus & 23 & $-9,-54,27$ & 4.83 & 7 \\
\hline
\end{tabular}

The absolute level of drift rate was associated, over and above the other parameters in the model, with interindividual differences in activity in the left inferior parietal lobule (IPL, BA 40; MNI coordinates: -39 -42 54), indicating that participants with higher drift rates show higher activity in IPL. Interindividual differences in boundary separation, on the other hand, were found to uniquely predict activation in the left striatum (putamen; -18 -6 15), indicating that participants with higher boundary parameters showed higher activity in the striatum. These correlations were not influenced by outliers, which the scatterplots in Figure 5 confirm. The nondecision parameter predicted individual differences in the left supramarginal gyrus (BA 40; $-45-3327$ ).

Even with lowered threshold ( $p<.005$, uncorrected), the interaction terms between Age and Time point on the one hand and the diffusion-model parameters on the other hand were not significant in the brain regions reported, supporting the generality of the observed effects. ROI analyses further demonstrate the similarity of these associations across age groups: When computing the correlation coefficients separately for the age groups, we find a significant correlation of percent signal changes in the striatum ROI and boundary parameter for both young $[r(24)=.44, p<.05]$ and old $[r(15)=.73, p<.01]$ participants. Similarly, we found a correlation of percent signal changes in the left IPL and drift parameter for both young $[r(24)=.51, p<.05]$ and old $[r(15)=.51, p=$ $.054]$ participants, although the correlation in the older participants was only marginally significant.

Because it has been hypothesized that the drift rate should be associated with brain areas that are responsible for stimulus-specific information accumulation (Heekeren et al., 2008), we additionally computed two separate models, one for the numerical task and one for the verbal task. When applying a more lenient threshold $(p<.001$, clus- ter of voxels $>10$ ), the resulting brain areas that correlated with the drift rate were the left inferior parietal lobe (BA 40; $-45-39-51$ ), the left middle occipital gyrus (BA 19; - $33-8130$ ), and the left superior temporal gyrus (BA 22; $-66-4215$ ) for the numerical task. For the verbal task, interindividual differences in drift rate predicted activity in the bilateral inferior parietal lobe (BA 40; -36 $-4254 ; 51-27$ 51) and left ventral premotor cortex (BA 6; $-57-336)$.

Finally, it can be predicted that the associations between individual differences in regional activity and individual differences in the boundary and drift rate estimates should generalize to individual differences in practice-related changes. To probe this prediction, we correlated changes (posttest - pretest) in the diffusion parameters to changes in the brain areas identified from the multiple regression analyses. In line with the positive level-level associations, these change-change associations had a positive direction (IPL drift rate: $r=.19, p=.248$; striatum boundary: $r=$ $.53, p<.001)$.

\section{DISCUSSION}

Perceptual decision-making performance (i.e., RT and accuracy) is a function of several underlying cognitive processes (e.g., Heekeren et al., 2008). In this study, we applied Ratcliff's diffusion model to deconstruct RT and accuracy data from a verbal and a numerical two-choice RT task into three separate parameters: the drift rate, indicating the accumulation rate of perceptual evidence (i.e., the efficiency of the decision process); the boundary separation, reflecting the more strategically influenced response criteria; and the nondecision time, combining the efficiency of sensory and motor aspect of performance. Interindividual differences in these parameters were then related to interindividual differences in brain activation as observed with 


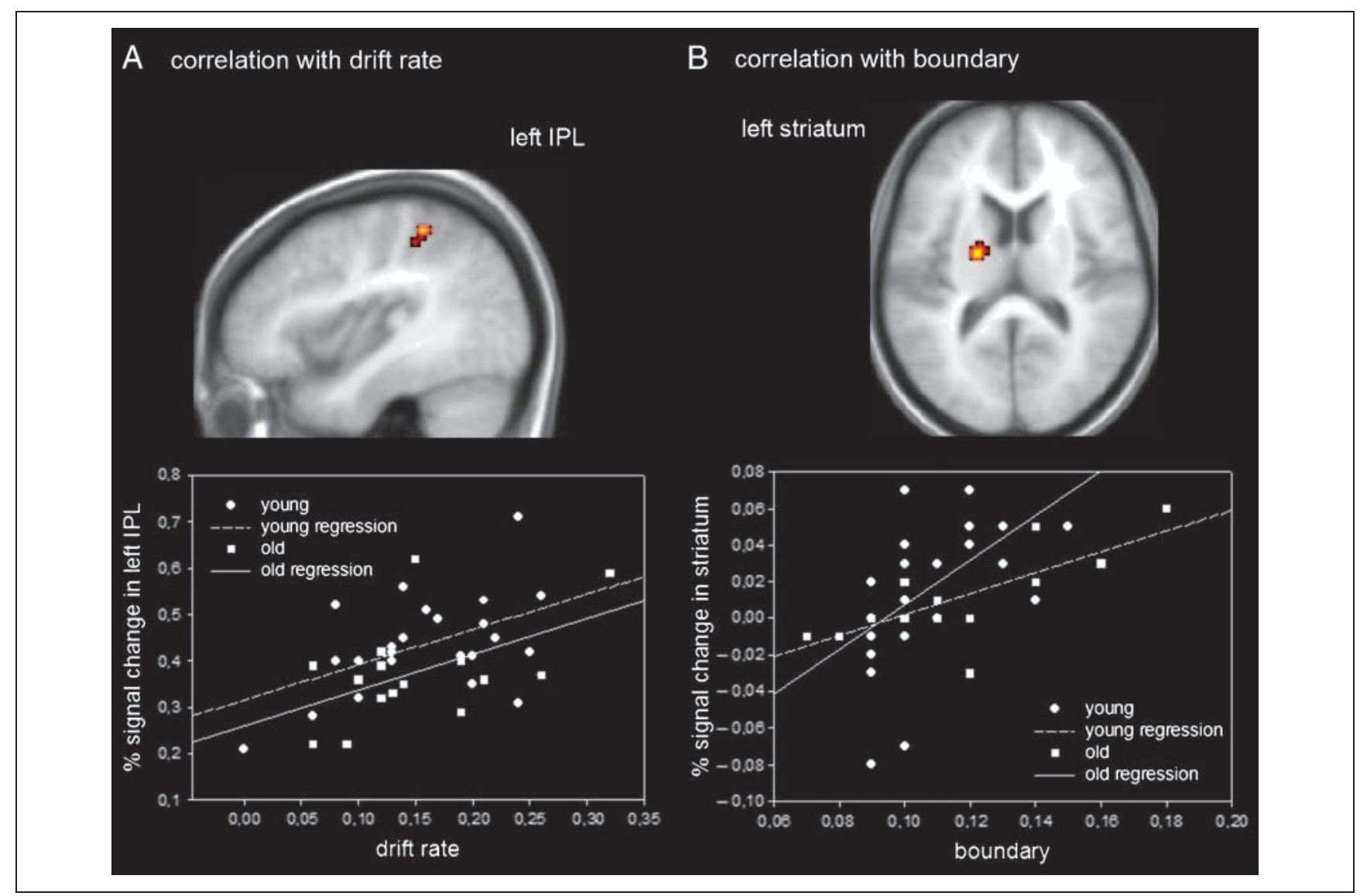

Figure 5. Activation map averaged over 39 subjects $(p<.05$, family-wise error corrected, $k>5)$ mapped onto an averaged image of the participants' normalized (MNI) T1-weighted images. (A) Brain activity (\% signal change averaged over pre- and posttraining sessions and over numerical and verbal tasks) correlated with the drift rate and (B) brain activity (\% signal change averaged over pre- and posttraining sessions and over numerical and verbal tasks) correlated with the boundary parameter. Note that the regression lines are plotted separately for old and young participants although there is no significant effect of age on the associations.

fMRI during performance of these tasks. In line with our hypotheses, we found that individuals with higher values of drift rate show stronger brain activity in areas that have been reported in processing of numbers and letters, namely, the left IPL, and that individuals with higher values in the boundary parameter showed a higher magnitude of striatal activity.

The parietal lobe, especially the horizontal segment of the intraparietal sulcus (HIPS), has been associated with number processing, and in particular, with the manipulation of numbers, such as in comparison or approximation (Dehaene et al., 2003). For example, patients with parietal lesions have been reported to suffer from difficulties in number processing (Dehaene, Dehaene-Lambertz, \& Cohen, 1998). Moreover, the parietal lobe contains an abstract representation of numerical magnitude independent of number notation (Eger et al., 2003). However, the left IPL has also been reported as being specific for single letter processing (Joseph et al., 2003, 2006; James et al., 2005). The fact that the left IPL is involved in both accumulation of numerical as well as letter information is supported by the consistent relation between the drift rate and the magnitude of activation in this region across separate analyses for the numerical and verbal task. Moreover, several studies have shown that activation in parietal regions correlates with successful memory, suggesting that the strength of activation might contribute to the eventual decision-making (Wheeler \& Buckner, 2003). Hence, parietal brain regions have been suggested to function as a mnemonic accumulator, accumulating signal until a criterion is reached and judgments are made, as described by signal-detection theory (Cabeza, Ciaramelli, Olson, \& Moscovitch, 2008; Wagner, Shannon, Kahn, \& Buckner, 2005). Our findings are in line with this evidence and suggest that the IPL might be involved in the accumulation of perceptual evidence-related letters and numbers.

The association between interindividual differences in the boundary parameter and the strength of activity in the striatum is consistent with at least two lines of previous research. First, it has been speculated that the basal ganglia are critical in speed-accuracy tradeoff (Bogacz \& Gurney, 2007; Lo \& Wang, 2006) by directly regulating the chain of neuronal responses leading to motor acts (Aron et al., 2003; Band \& van Boxtel, 1999; Mink, 1996). For example, the striatum has been linked to the suppression of movements during antisaccade tasks (Raemaekers et al., 2002) as well as 
to initiating movements in monkeys (Lebedev \& Nelson, 1999), therefore suggesting that the striatum is involved in both initiation and inhibition of motor responses. A second line of research has explored the neural correlates of speed-accuracy tradeoff by instructing participants to either respond as quickly as possible or as accurately as possible (Bogacz et al., 2010; Forstmann et al., 2008; Ivanoff et al., 2008; Van Veen et al., 2008). In terms of the diffusion model, such manipulations affect the boundary parameter of the decision process. When instructing participants to respond accurately, the boundaries are set wider. More information must thus be accumulated, assuming an unaltered drift rate, before a decision can be made, leading to more accurate and slower responses. In contrast, instructing participants to respond as fast as possible should lead to narrower boundaries. In accordance with our findings, Forstmann et al. (2008) report an association between the striatum and a caution parameter derived from a linear ballistic accumulator (LBA) model when comparing performance after speed and accuracy instruction. Likewise, Van Veen et al. (2008) report an association of basal ganglia activity with speed-accuracy tradeoff. But in both of these studies, higher activity in the striatum goes along with the speed compared to the accuracy instruction, whereas our results suggest enhanced striatal activity in relation to higher response caution. However, the activation reported by Forstmann et al. involved the caudate nucleus rather than the putamen (as in this study), whereas van Veen et al. report activity in both the caudate nucleus and the putamen. The present findings are, however, in line with findings of higher striatal activation during trials with a higher probability of response inhibition (Vink et al., 2005) when considering the boundary parameter as an indicator of cautious responding. In summary, the current evidence suggests that the striatum is involved in the regulation of response thresholds rather than, or at least in addition to, the initiation or suppression of responses. This is in line with a model of decision-making and response selection by Frank (2006) and Frank and Claus (2006) that conceptualizes the basal ganglia as a system that gates information flow from frontal cortex to the motor system. The circuit is assumed to facilitate or suppress action representations in frontal cortex. Two cell populations in the striatum are assumed to have opposing effects on the selection of an action. Activation in so-called "go" neurons facilitates the execution of an action considered, whereas activation in "no-go" neurons suppresses the competing responses, thereby constituting a gating mechanism in the striatum. Moreover, our findings are in line with the suggestion that failures in the fronto-striatal dopaminergic neural circuits underpin impulsive and compulsive acts (Fineberg et al., 2010).

As indicated by the absence of interactions between the observed associations (i.e., drift rate with the IPL and boundary separation with the striatum) and the factors Time point and Age, these associations show a fair amount of generalization over the adult lifespan and over different levels of task-specific expertise, despite the major effects these factors had on performance and the parameters of the diffusion model. Specifically, we observed higher drift rates and lower boundary parameters at posttest than at pretest, which replicates the findings of Dutilh, Vandekerckhove, Tuerlinckx, and Wagenmakers (2009, see also Ratcliff et al., 2006). Our data extend those previous findings by showing larger changes over time in these parameters for younger as compared to older adults. Consistent with the observed positive associations between individual differences in level of activity and level of the diffusion-model estimates, the corresponding associations between individual differences in changes also tended to go in the positive direction. In particular, the significant change-change correlation (postpretest) between brain activity in the striatum and the boundary parameter suggests that the striatum may also play a role in practice-related changes in boundary separation. Our finding that older adults have considerably longer nondecision times compared to young adults, on the other hand, replicate previous studies (Ratcliff et al., 2001, 2006; Ratcliff, Thapar, Gomez, et al., 2004; Ratcliff, Thapar, \& McKoon, 2004; Thapar, Ratcliff, \& McKoon, 2003). Finally, brain activation during performance of these tasks displayed changes over time: We found a decrease in task-related areas (left IFG, left IFJ, bilateral insula, dACC, right fusiform gyrus) and an increase in the so-called default-mode areas (vmPFC, precuneus, right STG). This pattern of changes is likely to reflect more automatic (i.e., less effortful) and perceptual expertise-like performance of the tasks at posttest (Kelly \& Garavan, 2005), but the mean level changes over time point are difficult to decisively interpret without an appropriate control group. We thus conclude that individual differences in striatal activity are related to criterion setting and that the rate of evidence accumulation for perceptual decisions of numerical and letter nature is related to stimulispecific brain regions in the parietal lobe.

\section{Acknowledgments}

Funded by the Max Planck Institute for Human Development, the Innovation Fund of the Max Planck Society, the Sofja Kovalevskaja Award (to M. L.) administered by the Alexander von Humboldt Foundation and donated by the German Federal Ministry for Education and Research (BMBF), Deutsche Forschungsgemeinschaft, and the BMBF. The work by the first author was supported by a post-doc grant of the Research Foundation-Flanders (FWOVlaanderen). We thank Colin Bauer, Annette Brose, Christian Chicherio, and all research assistants.

Reprint requests should be sent to Simone Kühn, Department of Experimental Psychology, University of Gent, Henri Dunantlaan 2, 9000 Gent, Belgium, or via e-mail: simone.kuhn@ugent.be or Martin Lövden, Department of Psychology, Lund University, Box 214, 22100 Lund, Sweden, or via e-mail: loevden@mpib-berlin. mpg.de.

\section{REFERENCES}

Aron, A. R., Schlaghecken, F., Fletcher, P. C., Bullmore, E. T., Eimer, M., Barker, R., et al. (2003). Inhibition of subliminally 
primed responses is mediated by the caudate and thalamus: Evidence from functional MRI and Huntington's disease. Brain, 126, 713-723.

Band, G. P., \& van Boxtel, G. J. (1999). Inhibitory motor control in stop paradigms: Review and reinterpretation of neural mechanisms. Acta Psychologica, 101, 179-211.

Bogacz, R., \& Gurney, K. (2007). The basal ganglia and cortex implement optimal decision making between alternative actions. Neural Computation, 19, 442-477.

Bogacz, R., Wagenmakers, E.-J., Forstmann, B. U., \& Nieuwenhuis, S. (2010). The neural basis of the speed-accuracy trade-off. Trends in Neurosciences, 33, $10-16$.

Brett, M., Anton, J.-C., Valabregue, R., \& Poline, J. B. (2002). Region of interest analysis using an SPM toolbox [abstract]. Presented at the 8th International Conference on Functional Mapping of the Human Brain, June 2-6.

Cabeza, R., Ciaramelli, E., Olson, I. R., \& Moscovitch, M. (2008). The parietal cortex and episodic memory: An attentional account. Nature Reviews Neuroscience, 9, 613-625.

Dehaene, S., Dehaene-Lambertz, G., \& Cohen, L. (1998). Abstract representation of numbers in the animal and human brain. Trends in Neurosciences, 21, 355-361.

Dehaene, S., Piazza, M., Pinel, P., \& Cohen, L. (2003). Three parietal circuits for number processing. Cognitive Neuropsychology, 20, 487-506.

Dutilh, G., Vandekerckhove, J., Tuerlinckx, F., \& Wagenmakers, E.-J. (2009). A diffusion model decomposition of the practice effect. Psychonomic Bulletin \& Review, 16, 1026-1036.

Eger, E., Sterzer, P., Russ, M. O., Giraud, A.-L., \& Kleinschmidt, A. (2003). A supramodal number representation in human intraparietal cortex. Neuron, 37, 719-725.

Fineberg, N. A., Potenza, M. N., Chamberlain, S. R., Berlin, H. A., Menzies, L., Bechara, A., et al. (2010). Probing compulsive and impulsive behaviors, from animal models to endophenotypes: A narrative review. Neuropsychopharmacology, 35, 591-604.

Forstmann, B. U., Dutilh, G., Brown, S., Neumann, J., von Cramon, D. Y., Ridderinkhof, K. R., et al. (2008). Striatum and pre-SMA facilitate decision-making under time pressure. Proceedings of the National Academy of Sciences, U.S.A., 105, 17538-17542.

Frank, M. J. (2006). Hold your horses: A dynamic computational role for the subthalamic nucleus in decision making. Neural Networks, 19, 1120-1136.

Frank, M. J., \& Claus, E. D. (2006). Anatomy of a decision: Striato-orbitofrontal interactions in reinforcement learning, decision making, and reversal. Psychological Review, 113, 300-326.

Gold, J. I., \& Shadlen, M. N. (2001). Neural computations that underlie decisions about sensory stimuli. Trends in Cognitive Sciences, 5, 10-16.

Heekeren, H. R., Marrett, S., \& Ungerleider, L. G. (2008). The neural systems that mediate human perceptual decision making. Nature Reviews Neuroscience, 9, 467-479.

Ivanoff, J., Branning, P., \& Marois, R. (2008). fMRI evidence for a dual process account of the speed-accuracy trade-off in decision-making. PloS One, 3, e2635.

James, K. H., James, T. W., Jobard, G., Wong, A. C.-N., \& Gauthier, I. (2005). Letter processing in the visual system: Different activation patterns for single letters and strings. Cognitive, Affective, \& Behavioral Neuroscience, 5, 452-466.

Joseph, J. E., Cerullo, M. A., Farley, A. B., Steinmetz, N. A., \& Mier, C. R. (2006). fMRI correlates of cortical specialization and generalization for letter processing. Neuroimage, 32, 806-820.

Joseph, J. E., Gathers, A. D., \& Piper, G. A. (2003). Shared and dissociated cortical regions for object and letter processing. Cognitive Brain Research, 17, 56-67.

Kelly, A. M., \& Garavan, H. (2005). Human functional neuroimaging of brain changes associated with practice. Cerebral Cortex, 15, 1089-1102.

Kim, J. N., \& Shadlen, M. N. (1999). Neural correlates of a decision in the dorsolateral prefrontal cortex of the macaque. Nature Neuroscience, 2, 176-185.

Lebedev, M. A., \& Nelson, R. J. (1999). Rhythmically firing neostriatal neurons in monkey: Activity patterns during reaction-time hand movements. Journal of Neurophysiology, 82, 1832-1842.

Lo, C. C., \& Wang, X. J. (2006). Cortico-basal ganglia circuit mechanism for a decision threshold in reaction time tasks. Nature Neuroscience, 9, 956-963.

Mason, M. F., Norton, M. I., van Horn, J. D., Wegner, D. M., Grafton, S. T., \& Macrea, C. N. (2007). Wandering minds: The default network and stimulus-independent thought. Science, 315, 393-395.

Mink, J. W. (1996). The basal ganglia: Focused selection and inhibition of competing motor programs. Progress in Neurobiology, 50, 381-425.

Raemaekers, M., Jansma, J. M., Cahn, W., van der Geest, J. N., van der Linden, J. A., Kahn, R. S., et al. (2002). Neuronal substrate of the saccadic inhibition deficit in schizophrenia investigated with 3-dimensional event-related functional magnetic resonance imaging. Archives of General Psychiatry, 59, 313-320

Raichle, M. E., MacLeod, A. M., Snyder, A. Z., Powers, W. J., Gusnard, D. A., \& Shulman, G. L. (2001). A default mode of brain function. Proceedings of the National Academy of Sciences, U.S.A., 98, 676-682.

Ratcliff, R. (1978). A theory of memory retrieval. Psychological Review, 85, 59-108.

Ratcliff, R. (2002). A diffusion model account of response time and accuracy in a brightness discrimination task: Fitting real data and failing to fit fake but plausible data. Psychonomic Bulletin \& Review, 9, 278-291.

Ratcliff, R., Cherian, A., \& Segraves, M. A. (2003). A comparison of macaque behavior and superior colliculus neuronal activity to predictions from models of two-choice decisions. Journal of Neurophysiology, 90, 1392-1407.

Ratcliff, R., Hasegawa, Y. T., Hasegawa, R. P., Smith, P. L., \& Segraves, M. A. (2007). Dual diffusion model for single cell recording data from the superior colliculus in a brightness-discrimination task. Journal of Neurophysiology, 97, 1756-1774.

Ratcliff, R., \& Rouder, J. N. (2000). A diffusion model account of masking in letter identification. Journal of Experimental Psychology: Human Perception and Performance, 26, 127-140.

Ratcliff, R., Thapar, A., Gomez, P., \& McKoon, G. (2004). A diffusion model analysis of the effects of aging in the lexical-decision task. Psychology and Aging, 19, 278-289.

Ratcliff, R., Thapar, A., \& McKoon, G. (2001). The effects of aging on reaction time in a signal detection task. Psychology and Aging, 16, 323-341.

Ratcliff, R., Thapar, A., \& McKoon, G. (2004). A diffusion model analysis of the effects of aging on recognition memory. Journal of Memory and Language, 50, 408-424.

Ratcliff, R., Thapar, A., \& McKoon, G. (2006). Aging, practice, and perceptual tasks: A diffusion model analysis. Psychology of Aging, 21, 353-371.

Ratcliff, R., \& Tuerlinckx, F. (2002). Estimating the parameters of the diffusion model: Approaches to dealing with 
contaminant reaction times and parameter variability. Psychonomic Bulletin \& Review, 9, 438-481.

Schmiedek, F., Bauer, C., Lövdén, M., Brose, A., \& Lindenberger, U. (2010). Cognitive enrichment in old age: Web-based training programs. GeroPsych, 23, 59-67.

Schmiedek, F., Lövdén, M., \& Lindenberger, U. (2010). Hundred days of cognitive training enhance broad cognitive abilities in adulthood: Findings from the COGITO study. Frontiers in Aging Neuroscience, 2, doi: 10.3389/fnagi.2010.00027.

Smith, P. L., \& Ratcliff, R. (2004). Psychology and neurobiology of simple decisions. Trends in Neuroscience, 27, 161-168.

Starns, J. J., \& Ratcliff, R. (2010). The effects of aging on the speed-accuracy compromise: Boundary optimality in the diffusion model. Psychology and Aging.

Thapar, A., Ratcliff, R., \& McKoon, G. (2003). A diffusion model analysis of the effects of aging on letter discrimination. Psychology and Aging, 18, 415-429.
Van Veen, V., Krug, M. K., \& Carter, C. S. (2008). The neural and computational basis of controlled speed-accuracy trade-off during task performance. Journal of Cognitive Neuroscience, 20, 1952-1965.

Vink, M., Kahn, R. S., Raemaekers, M., van den Heuvel, M., Boersma, M., \& Ramsey, N. F. (2005). Function of striatum beyond inhibition and execution of motor responses.

Human Brain Mapping, 25, 336-344.

Wagner, A. D., Shannon, B. J., Kahn, I., \& Buckner, R. L. (2005). Parietal lobe contributions to episodic memory retrieval. Trends in Cognitive Sciences, 9, 445-453.

Wheeler, M. E., \& Buckner, R. L. (2003). Functional dissociation among components of remembering: Control, perceived oldness, and content. Journal of Neuroscience, 23, 3869-3880

Wickelgren, W. A. (1977). Speed-accuracy trade-off and information processing dynamics. Acta Psychologica, 41, $67-85$. 